Pacific Journal of Mathematics

ON A THEOREM OF MERGELYAN 


\title{
ON A THEOREM OF MERGELYAN
}

\author{
JOHN GARNETT
}

The following is a theorem of S. N. Mergelyan.

Theorem. If $X$ is a compact plane set with finitely many complementary components, then every continuous function on $X$ which is analytic on the interior is a uniform limit on $X$ of rational functions.

Here two short proofs of the theorem are given. Both proofs are obtained by considering the measures on $X$ orthogonal to the algebra of rational functions.

Let $X$ be a compact plane set and suppose its complement $C \backslash X$ has finitely many components. In 1951 Mergelyan [9] proved:

Mergelyan's Theorem. Every function continuous on $X$ and analytic on the interior is uniformly approximable by rational functions.

By examining the measures on $X$ orthogonal to the rational functions, Glicksberg and Wermer [6] have found an elegant proof of Mergelyan's Theorem in the case $C \backslash X$ connected. Their argument has recently been extended by Ahern and Sarason [1] and by Glicksberg [5] to give functional analytic proofs of the general Mergelyan result. However those proofs are quite long. Here we give two shorter proofs of the theorem. The first proof begins with the known fact that the question of approximation is a local one, and the theorem is thereby reduced to the simply connected case. The second proof follows the reasoning of [6]. Here we use some results from [1] and [5], but avoid the more lengthy arguments in those papers. Though longer than the first, this second proof is included because the ideas therein yield related results not accessible via our first proof.

Denote by $A(X)$ the Banach algebra of functions continuous on $X$ and analytic on $X^{0}$, the interior. Let $R(X)$ be the closed subalgebra of $A(X)$ spanned by the rational functions analytic on $X$. By a measure on $X$ we mean a finite complex Borel measure on $X$. Write $\mu \perp R(X)$ is $\mu$ is a measure on $X$ orthogonal to $R(X)$, and $\mu \perp A(X)$ if $\mu$ annihilates $A(X)$ as well. As have several previous authors, we prove the Mergelyan Theorem by showing $\mu \perp R(X)$ implies $\mu \perp A(X)$.

2. The first proof. We quote the following lemma of Bishop [3, p. 40]. This lemma was stated polynomials, but Bishop's proof is valid for rational functions. 
Lemma 2.1. (Bishop). Let $X$ be a compact subset of $C$. For $x$ a real number, set

$$
L_{x}=X \cap\{z: \text { Real } z \leqq x\}, R_{x}=X \cap\{z: \text { Real } z \geqq x\} .
$$

Let $\mu \perp R(X)$. Then for almost all $x$ there are measures $\nu_{1}$ on $L_{x}$ and $\nu_{2}$ on $R_{x}$ such that $\nu_{1} \perp R\left(L_{x}\right), \nu_{2} \perp R\left(R_{x}\right)$ and $\mu=\nu_{1}+\nu_{2}$.

This lemma gives the known result that questions concerning $R(X)$ are local ones.

CoRollary 2.2. Let $f$ be a continuous function on the compact set $X$. Assume each point in $X$ has a closed neighborhood $K$ such that the restricted function $f \mid K$ is in $R(K)$. Then $f$ is in $R(X)$.

Proof. Let $\mu$ be a measure orthogonal to $R(X)$ and let $\left\{U_{1}, U_{2}\right.$, $\left.\cdots, U_{n}\right\}$ be any finite open cover of $X$. It follows from repeated applications of 2.1 that there are finitely many compact rectangles $\left\{T_{1}, \cdots, T_{m}\right\}$ and measures $\nu_{j}$ on $T_{j}$ such that

(i) $T_{j} \subset U_{i}$ for some $i$

(ii) $\nu_{j} \perp R\left(T_{j}\right)$

(iii) $\mu=\sum_{j=1}^{m} \nu_{j}$.

If $f$ satisfies the hypotheses, then using such a decomposition we see that $\int f d \mu=0$. This implies $f \in R(X)$.

Actually we can localize measures as in the proof of 2.2 without appealing to Bishop's lemma. Instead we use an observation shown us by A. Browder, who credits it to K. Hoffman. For a measure $\mu$ on $X$ define its Cauchy transform

$$
\widehat{\mu}(\alpha)=\int(z-\alpha)^{-1} d \mu(z) \text {. }
$$

Then $\hat{\mu}$ is locally integrable with respect to plane Lebesgue measure. Also $\hat{\mu}=0$ almost everywhere if and only if $\mu=0$. See [11]. The observation, whose proof we only sketch, is this:

(a) $\mu \perp R(X)$ if and only if $\hat{\mu}=0$ on $C \backslash X$. This is seen by taking partial fractions and by "pushing poles together."

(b) An application of Green's theorem yields, for $\phi$ a $C^{\infty}$ function with compact support,

$$
\varphi \hat{\mu}=\widehat{\varphi \mu}+\hat{\sigma} \quad \text { where } \quad \sigma=\frac{1}{2 \pi i} \frac{\partial \varphi}{\partial \bar{z}} \hat{\mu}(z) d z \wedge d \bar{z} .
$$

Now if $\mu \perp R(X)$ and $K$ is the closed support of $\varphi$, then by (a) and (b),

$$
\varphi \mu+\sigma \perp R(X \cap K) \text {. }
$$


Letting $\varphi$ run through a finite partition of unity $\left\{\varphi_{j}\right\}_{j=1}^{m}$ and setting $\nu_{j}=\varphi_{j} \mu+1 / 2 \pi i \partial \varphi_{j} / \partial \bar{z} \hat{\mu}(z) d z \wedge d \bar{z}$ we obtain a decomposition of $\mu$ satisfying (i), (ii) and (iii) in the proof of 2.2 .

Proof of theorem. Let $H_{1}, H_{2}, \cdots, H_{n}$ be the bounded components of $C \backslash X$ and let $f \in A(X)$. For each point in $X$ choose a compact neighborhood $K$ with diameter less than the diameter of each $H_{j}$. Then $C \backslash K$ is connected and $f \mid K$ is in $A(K)$. Thus by [6], $f \mid K$ is in $R(K)$. Hence $f$ is in $R(X)$ by 2.2 .

We note that this proof is still valid when $C \backslash X$ has infinitely many components but the diameters of these components are bounded below. Examples of such sets are easily constructed.

3. The second proof. By the maximum modulus principle $A(X)$ can be identified with the algebra of boundary values of functions in $A(X)$. We will view $A(X)$ as a closed algebra of continuous functions in $\partial X$, the boundary of $X$, and we consider now only measures supported on $\partial(X)$.

We begin with a theorem of Walsh [10] and Lebesgue [8]. A more recent proof is contained in the first three lemmas of [4]. $C^{R}(\partial X)$ is the space of real continuous functions on $\partial X$, and Real $R(X)$ is the space (restrictions to $\partial X$ ) of real parts of functions in $R(X)$.

Walsh-Lebesgue Theorem. Let $H_{1}, H_{2}, \cdots, H_{n}$ be the bounded components of $C \backslash X$ and let $a_{j} \in H_{j} . \quad$ Then Real $R(X) \oplus \operatorname{Span}\left\{\log \left|z-a_{j}\right|\right.$ : $1 \leqq j \leqq n\}$ is uniformly dense in $C^{R}(\partial X)$.

This means the space of real measures on $\partial X$ orthogonal to $R(X)$ has dimension at most $n$. It also means the Dirichlet problem is solvable on $X$; if $u \in C^{R}(\partial X)$ then there is a unique $\hat{u}$ in $C^{R}(X)$ harmonic on $X^{0}$ with $u=\hat{u}$ on $\partial X$. Hence for $z \in X$ there is a unique probability measure $\lambda_{z}$ on $\partial X$ such that $\hat{u}(z)=\int_{\partial X} u d \lambda_{z}$ for all $u \in C^{R}(\partial X)$ It follows directly that $\lambda_{z}$ is a representing measure for $A(X)$, as $\lambda_{z}$ is positive and

$$
f(z)=\int_{\partial X} f d \lambda_{z} \text { for all } f \in A(X) .
$$

It also follows that $\lambda_{z}$ is an Arens-Singer measure for $A(X)$,

$$
\log |f(z)|=\int_{\partial X} \log |f| d \lambda_{z} \quad \text { for } f \in A(X) \text { and } \frac{1}{f} \in A(X) .
$$

Moreover the Walsh-Lebesgue theorem implies that $\lambda_{z}$ is the unique 
Arens-Singer measure for $R(X)$. Consequently the assumptions of the paper [1] of Ahern and Sarason apply to $R(X)$. We now state a lemma of theirs and some immediate consequences. All except Corollary 3.4 are proven on pp. 126-128 of [1].

LEMma 3.1. (Ahern-Sarason). Let $\left(v_{n}\right)_{n=1}^{\infty}$ be a sequence of nonnegative functions in $C^{R}(\partial X)$ with $\int v_{n} d \lambda_{z} \rightarrow 0$. Then there is a subsequence $\left(u_{n}\right)_{n=1}^{\infty}$ and a sequence $\left(f_{n}\right)_{n=1}^{\infty}$ of functions in $R(X)$ such that $\left|f_{n}\right| \leqq e^{-u_{n}}$ and $f_{n} \rightarrow 1$ almost everywhere $\lambda_{z}$.

Corollary 3.2. (F. and M. Riesz Theorem). If $z \in X$ and $\mu \perp R(X)$, let $\mu=\mu_{z}+\tilde{\mu}_{z}$ be the Lebesgue decomposition of $\mu$ with respect to $\lambda_{z}$. Then $\mu_{z} \perp R(X)$ and $\tilde{\mu}_{z} \perp R(X)$.

CoRollaRY 3.3. For $z \in X$, every representing measure on $\partial X$ for $z$ is absolutely continuous with respect to $\lambda_{z}$. In particular, when $z \in \partial X$, the only representing measure is the point mass at $z$.

The next corollary is a consequence of the F. and M. Riesz theorem, and is Theorem 2.8 of Glicksberg's paper [5].

CoROLlary 3.4. If $\mu \perp R(X)$, then there is a sequence $\left(z_{n}\right)_{n=1}^{\infty}$ in $X$ such that

$$
\mu=\mu_{s}+\sum_{n=1}^{\infty} \mu_{z_{n}}
$$

where the series converges in norm, each term is orthogonal to $R(X)$, $\mu_{z_{n}}$ is absolutely continuous with respect to $\lambda_{z_{n}}$ and $\mu_{8}$ is singular with respect to all representing measures.

Proof. First take the "simultaneous Lebesgue decomposition of $\mu$ with respect to all $\lambda_{z} . "$ That is, let $c=\sup \left\{\left\|\mu_{z}\right\|: z \in X\right\}$ and choose $z_{1}$ so that $\left\|\mu_{z_{1}}\right\|>c / 2$. Take the Lebesgue decomposition $\mu=\tilde{\mu}_{z_{1}}+\mu_{z_{1}}$. Repeating the argument with $\tilde{\mu}_{z_{1}}$ and continuing by induction yields a decomposition (1), in which $\mu_{s}$ is singular with respect to all $\lambda_{z}$.

By Corollary 3.2, $\mu_{z_{1}} \perp R(X)$, and so by induction every $\mu_{z_{n}}$ (and hence $\mu_{s}$ as well) is ortogonal to $R(X)$. Finally by Corollary 3.3, $\mu_{s}$ is singular with respect to every representing measure.

From Corollary 3.4 we see that to show any $\mu \in(R(X))^{\perp}$ annihilates $A(X)$ it suffices to consider the case when $\mu=\mu_{z}$ for some $z$ and the case when $\mu$ is singular with respect to all representing measures. The latter case is settled by the following argument of Wilken [12], 
valid for any compact plane set.

Lemma 3.5. (Wilken). Let $X$ be any compact plane set and let $\mu \perp R(X)$. If $\mu$ is singular with respect to all representing measures then $\mu=0$.

Proof. As in [12], there is a point $z \in X$ such that

$$
\int \frac{d|\mu|(\zeta)}{|z-\zeta|}<\infty \text { and } \int \frac{d \mu(\zeta)}{z-\zeta}=c \neq 0 \text {. }
$$

Then the measure $\nu=1 / c \mu(\zeta) / z-\zeta$ is a complex measure representing $z$; so that by [7] there is a positive representing measure $m$ for $z$ absolutely continuous with respect to $|\nu|$. Then $m$ is absolutely continuous with respect to $|\mu|$ and the assertion obtains.

For our analysis of the absolutely continuous orthogonal measures we need two more lemmas. For $z \in X$, write $\lambda=\lambda_{z}$, and let $H^{2}(R(X))$ be the closure of $R(X)$ in $L^{2}(\lambda)$. Set $H^{\infty}(R(X))=H^{2}(R(X)) \cap L^{\infty}(\lambda)$. Define $H^{2}(A(X))$ and $H^{\infty}(A(X))$ analogously. The first lemma is from Ahern and Sarason [1] and follows directly from Lemma 3.1 by an argument of Hoffman and Wermer. (See also [5] and [11].)

LEMMA 3.6. If $h$ is in $H^{\infty}(R(X))$, then there is a sequence $\left(h_{n}\right)_{n=1}^{\infty}$ in $R(X)$ with $\left\|h_{n}\right\|_{\infty} \leqq\|h\|_{\infty}$ and $h_{n} \rightarrow h$ almost everywhere $\lambda$.

The second lemma is a minor variation of Glicksberg's Lemma 3.16 of [5]. The proof is due to T. W. Gamelin and the author.

\section{Lemma 3.7. $H^{2}(R(X))=H^{2}((A(X))$.}

Proof. Let $\overline{H_{0}^{2}(R(X))}$ be the space of complex conjugates of functions in $H^{2}(R(X))$ having integral zero. Since $\lambda$ is multiplicative on $R(X), L^{2}(\lambda)$ has the following orthogonal decomposition:

$$
L^{2}(\lambda)=H^{2}(R(X)) \oplus \overline{H_{0}^{2}(R(X))} \oplus E .
$$

The space $E$ is spanned by the real functions in $L^{2}$ annihilating $R(X)$ and thus by the Walsh-Lebesgue theorem $E$ is finite dimesional. Also $\lambda$ is multiplicative on $A(X)$, so an orthogonality argument gives

$$
H^{2}(R(X)) \subset H^{2}(A(X)) \subset H^{2}(R(X)) \oplus E .
$$

This means $H^{2}(A(X))=H^{2}(R(X)) \oplus N$ where $N$ is finite dimensional. Let $G_{1}, G_{2}, \cdots, G_{p}$ be an orthonormal basis of $N$ and for an appropriately small $\varepsilon$ choose $g_{1}, g_{2}, \cdots, g_{p}$ in $A(X)$ with $\left\|g_{j}-G_{j}\right\|_{2}<\varepsilon$. Let $J$ be the span of $\left\{g_{1}, \cdots, g_{p}\right\}$. Then 


$$
H^{2}(A(X))=H^{2}(R(X))+J
$$

where the sum is direct but not necessarily orthogonal. Let $P_{J}$ be the projection of $H^{2}(A(X))$ onto $J$ which is zero on $H^{2}(R(X))$ For $f \in R(X)$ and $g \in J$ define $T_{f}(g)=P_{J}(f g)$. Then $T$ is a representation of $R(X)$ on $J$. Since $R(X)$ is commutative and $J$ is finite dimensional there is a common eigenvector $g \in J$. Therefore for $f \in R(X)$ we have

$$
f g=\varphi(f) \cdot g+r
$$

where $r \in H^{2}(R(X))$ and $\phi(f) \in \boldsymbol{C}$.

Clearly $\varphi$ is a multiplicative linear functional on $R(X)$, so that there is a point $z_{1} \in X$ such that $\varphi(f)=f\left(z_{1}\right)$ for all $f \in R(X)$. Also because $g$ is bounded $\varphi$ extends continuously to $H^{2}(R(X))$ and so there is an $F \in L^{2}(\lambda)$ such that $f\left(z_{1}\right)=\int f F d \lambda$. Hence by (7) $z_{1}$ has a positive representing measure absolutely continuous with respect to $\lambda$. By Corollary 3.3 then $z_{1} \notin \partial X$.

Taking $f=z$ in (2), we have $h=\left(z-z_{1}\right) g \in H^{2}(R(X))$ Hence there is a sequence $\left(h_{n}\right)_{n=1}^{\infty}$ in $R(X)$ converging to $h$ in $L^{2}(\lambda)$. Let $c=$ $\int h F d \lambda=\lim _{n \rightarrow \infty} \int h_{n} F d \lambda$. Then since $z_{1} \in X^{0}, r_{n}=h_{n}-h_{n}\left(z_{1}\right) / z-z_{1}$ is in $R(X)$ and $r_{n}$ converges to $g-c\left(z-z_{1}\right)^{-1}$ in $L^{2}(\lambda)$. Thus

$$
g-c\left(z-z_{1}\right)^{-1} \in H^{2}(R(X)) \text {. }
$$

If $c=0$, then $g \in H^{2}(R(X))$ and we are done. On the other hand if $c \neq 0$, then $\left(z-z_{1}\right)^{-1} \in H^{2}(A(X))$. Being bounded on $\partial X,\left(z-z_{1}\right)^{-1}$ is therefore in $H^{\infty}(A(X))$. Now $H^{\infty}(A(X))$ is an algebra, and consequently $\left(z-z_{1}\right)^{-n} \in H^{2}(A(X))$ for all $n$. But $H^{2}(R(X))$ has finite codimension in $H^{2}(A(X))$; whence some polynomial in $\left(z-z_{1}\right)^{-1}$ is in $H^{2}(R(X))$. That is

$$
\frac{a_{1}}{z-z_{1}}+\frac{a_{2}}{\left(z-z_{1}\right)^{2}}+\cdots+\frac{a_{n}}{\left(z-z_{1}\right)^{n}} \in H^{2}(R(X)) . \quad\left(a_{n} \neq 0\right) .
$$

Multiplying by $\left(z-z_{1}\right)^{n-1}$ we have $\left.\left(z-z_{1}\right)^{-1} \in H^{2}(R X)\right)$. By (3), then $g \in H^{2}(R(X))$. Therefore $J=\{0\}$ and

$$
H^{2}(R(X))=H^{2}(A(X)) \text {. }
$$

To conclude the proof of Mergelyan's theorem, let $\mu \perp R(X)$. We can assume $\mu$ is absolutely continuous with respect to some $\lambda_{z}$. Let $h \in A(X)$. Then by $3.7 h \in H^{2}(R(X))$ and so by 3.6 there is a sequence $\left(h_{n}\right)_{n=1}^{\infty}$ in $R(X)$ converging boundedly pointwise to $h$ almost everywhere $\lambda_{z}$. As $\int h_{n} d \mu=0$, dominated convergence implies $\int h d \mu=0$ and $\mu$ is orthogonal to $A(X)$. 
We should remark that the proof of Lemma 3.7 actually shows that $H^{\infty}(R(X))$ is maximal among the subalgebras of $L^{\infty}(\lambda)$ on which $\lambda$ is multiplicative. This means that one can compute the defect of Real $R(X)$ in $C^{R}(\partial X)$ and obtain the other results that Ahern and Sarason do in [2] without using the deeper theorems of their earlier paper [1].

Added in proof. After a closer reading of the literature we have found that the proof of Mergelyan's theorem in $\S 2$ was essentially given by Laura Kodama in "Boundary measures of analytic differentials" Pacific J. Math., 15 (1965), 1261-1277.

We wish to thank T. W. Gamelin and K. Hoffman for helpful conversations.

\section{REFERENCES}

1. P.R. Ahern and Donald Sarason, The $H^{p}$ spaces of a class of function of algebras, Acta. Math. 117 (1967), 123-163.

2. P. R. Ahern and Donald Sarason, On some hypodichlet algebras of analytic functions, Amer. J. Math. 89 (1967), 932-941.

3. E. Bishop, Subalgebras of functions on a Riemann surface, Pacific J. Math. 8 (1958), 29-50.

4. L. Carleson, Mergelyan's theorem on uniform polynomial approximation, Math. Scand. 15 (1964), 167-175.

5. I. Glicksberg, Dominant representing measures and rational approximation Trans. Amer. Math. Soc. 130 (1968), 425-462.

6. I. Glicksberg and J. Wermer, Measures orthogonal to a Dirichlet algebra, Duke Math. J. 30 (1963), 661-666.

7. K. Hoffman and H. Rossi, On the extension of positive weak continuous functionals, Duke Math. J. 34 (1967), 453-466.

8. H. Lebesgue, Sur le problem de Dirichlet, Rend. Palmero, 29 (1907), 371-402.

9. S. N. Mergelyan, Uniform approximation to functions of a complex variable, Amer. Math. Soc. Translation No. 101.

10. J. L. Walsh, The approximation of harmonic functions by harmonic polynomials and harmonic rational functions, Bull. Amer. Math. Soc. 35 (1929), 499-544.

11. J. Wermer, Seminar über Funktionen-Algebren, Springer, Berlin, 1964.

12. D. R. Wilken, Lebesgue measure for parts of $R(X)$, Proc. Amer. Math. Soc. 18 (1967), 508-512.

Received May 25, 1967. Research sponsored by the Air Force Office of Scientific Research, Office of Aerospace Research, United States Air Force, under AFOSR Grant No. 335-63. 



\section{PACIFIC JOURNAL OF MATHEMATICS}

\section{EDITORS}

\section{H. ROYDEN}

Stanford University

Stanford, California

R. R. Phelps

University of Washington

Seattle, Washington 98105

\section{J. DugundJI}

Department of Mathematics

University of Southern California

Los Angeles, California 90007

RICHARD ARENS

University of California

Los Angeles, California 90024

\section{ASSOCIATE EDITORS}
E. F. BeCKENBACH
B. H. NeumanN
F. WOLF
K. YosIDA

\section{SUPPORTING INSTITUTIONS}

\author{
UNIVERSITY OF BRITISH COLUMBIA \\ CALIFORNIA INSTITUTE OF TECHNOLOGY \\ UNIVERSITY OF CALIFORNIA \\ MONTANA STATE UNIVERSITY \\ UNIVERSITY OF NEVADA \\ NEW MEXICO STATE UNIVERSITY \\ OREGON STATE UNIVERSITY \\ UNIVERSITY OF OREGON \\ OSAKA UNIVERSITY \\ UNIVERSITY OF SOUTHERN CALIFORNIA
}

\author{
STANFORD UNIVERSITY \\ UNIVERSITY OF TOKYO \\ UNIVERSITY OF UTAH \\ WASHINGTON STATE UNIVERSITY \\ UNIVERSITY OF WASHINGTON \\ AMERICAN MATHEMATICAL SOCIETY \\ CHEVRON RESEARCH CORPORATION \\ TRW SYSTEMS \\ NAVAL WEAPONS CENTER
}

Mathematical papers intended for publication in the Pacific Journal of Mathematics should be in typed form or offset-reproduced, double spaced with large margins. Underline Greek letters in red, German in green, and script in blue. The first paragraph or two must be capable of being used separately as a synopsis of the entire paper. It should not contain references to the bibliography. Manuscripts, in duplicate if possible, may be sent to any one of the four editors. All other communications to the editors should be addressed to the managing editor, Richard Arens, University of California, Los Angeles, California 90024.

Each author of each article receives 50 reprints free of charge; additional copies may be obtained at cost in multiples of 50 .

The Pacific Journal of Mathematics is published monthly. Effective with Volume 16 the price per volume (3 numbers) is $\$ 8.00$; single issues, $\$ 3.00$. Special price for current issues to individual faculty members of supporting institutions and to individual members of the American Mathematical Society: $\$ 4.00$ per volume; single issues $\$ 1.50$. Back numbers are available.

Subscriptions, orders for back numbers, and changes of address should be sent to Pacific Journal of Mathematics, 103 Highland Boulevard, Berkeley 8, California.

Printed at Kokusai Bunken Insatsusha (International Academic Printing Co., Ltd.), 7-17, Fujimi 2-chome, Chiyoda-ku, Tokyo, Japan.

PUBLISHED BY PACIFIC JOURNAL OF MATHEMATICS, A NON-PROFIT CORPORATION

The Supporting Institutions listed above contribute to the cost of publication of this Journal, but they are not owners of publishers and have no responsibility for its content or policies. 


\section{Pacific Journal of Mathematics}

\section{Vol. 26, No. $3 \quad$ BadMonth, 1968}

Leonard Asimow, Universally well-capped cones ................. 421

Lawrence Peter Belluce, William A. Kirk and Eugene Francis Steiner,

Normal structure in Banach spaces ..................... 433

William Jay Davis, Bases in Hilbert space.................... 441

Larry Lee Dornhoff, p-automorphic p-groups and homogeneous

algebras..................................... 447

William Grady Dotson, Jr. and W. R. Mann, A generalized corollary of the

Browder-Kirk fixed point theorem ....................... 455

John Brady Garnett, On a theorem of Mergelyan ................. 461

Matthew Gould, Multiplicity type and subalgebra structure in universal

algebras............................................. 469

Marvin D. Green, A locally convex topology on a preordered space . . . . . 487

Pierre A. Grillet and Mario Petrich, Ideal extensions of semigroups . . . . . . 493

Kyong Taik Hahn, A remark on integral functions of several complex

variables ... . . . . . . . . . . . . . . . . . . . . . . . . . . . . . . . . . . . 509

Choo Whan Kim, Uniform approximation of doubly stochastic

operators..................................... 515

Charles Alan McCarthy and L. Tzafriri, Projections in $\mathscr{L}_{1}$ and

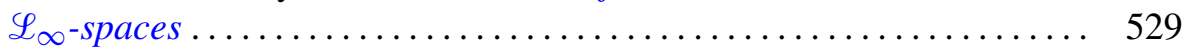

Alfred Berry Manaster, Full co-ordinals of RETs ................ 547

Donald Steven Passman, $p$-solvable doubly transitive permutation

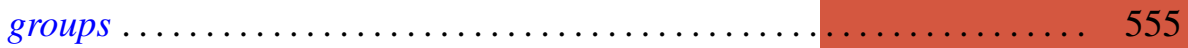

Neal Jules Rothman, An $L^{1}$ algebra for linearly quasi-ordered compact

semigroups ....................................... 579

James DeWitt Stein, Homomorphisms of semi-simple algebras .......... 589

Jacques Tits and Lucien Waelbroeck, The integration of a Lie algebra

representation ...............................

David Vere-Jones, Ergodic properties of nonnegative matrices. II ........ 601

Donald Rayl Wilken, The support of representing measures for $R(X) \ldots \ldots 621$

Abraham Zaks, Simple modules and hereditary rings .... . . 